\title{
Erratum: Collective modes and gapped momentum states in liquid Ga: Experiment, theory, and simulation [Phys. Rev. B 101, 214312 (2020)]
}

\author{
R. M. Khusnutdinoff, C. Cockrell ${ }^{\circ}$, O. A. Dicks, A. C. S. Jensen, M. D. Le, L. Wang, M. T. Dove, \\ A. V. Mokshin, V. V. Brazhkin, and K. Trachenko
}

(Received 26 July 2020; revised 20 November 2020; published 9 March 2021)

DOI: 10.1103/PhysRevB.103.099901

We would like to correct the theoretical section (Sec. IV) of our original paper and, in particular, our reference to equations used in that section to calculate theoretical current spectra. The equations used were from our earlier theory in Refs. [1-5] (Refs. [32-36] in our original paper), although the equations referred to originally were carried over from an earlier version of the paper in error as noted in Ref. [6]. The experimental and modeling parts of the paper are unaffected. The correction to the theoretical section (Sec. IV) does not affect any of our results and conclusions. For clarity, we reproduce the theoretical equations we used from our previous papers [1-5] below in full, as is done in the corrected paper [7].

\section{COLLECTIVE EXCITATIONS AND DISPERSION RELATION}

Using the memory function formalism in the self-consistent relaxation theory [1-5], the dynamic structure factor $S(k, \omega)$ is

$$
S(k, \omega)=\frac{S(k)}{2 \pi} \frac{\Delta_{1}^{2}(k) \Delta_{2}^{2}(k) \Delta_{3}^{2}(k)}{\Delta_{4}^{2}(k)-\Delta_{3}^{2}(k)} \frac{\left[4 \Delta_{4}^{2}(k)-\omega^{2}\right]^{1 / 2}}{\omega^{6}+\mathcal{A}_{1}(k) \omega^{4}+\mathcal{A}_{2}(k) \omega^{2}+\mathcal{A}_{3}(k)},
$$

where

$$
\begin{aligned}
& \mathcal{A}_{1}(k)=\frac{\Delta_{3}^{4}(k)-\Delta_{2}^{2}(k)\left[2 \Delta_{4}^{2}(k)-\Delta_{3}^{2}(k)\right]}{\Delta_{4}^{2}(k)-\Delta_{3}^{2}(k)}-2 \Delta_{1}^{2}(k), \\
& \mathcal{A}_{2}(k)=\frac{\Delta_{2}^{4}(k) \Delta_{4}^{2}(k)-2 \Delta_{1}^{2}(k) \Delta_{3}^{4}(k)+\Delta_{1}^{2}(k) \Delta_{2}^{2}(k)\left[2 \Delta_{4}^{2}(k)-\Delta_{3}^{2}(k)\right]}{\Delta_{4}^{2}(k)-\Delta_{3}^{2}(k)}+\Delta_{1}^{4}(k), \\
& \mathcal{A}_{3}(k)=\frac{\Delta_{1}^{4}(k) \Delta_{3}^{4}(k)}{\Delta_{4}^{2}(k)-\Delta_{3}^{2}(k)} .
\end{aligned}
$$

Here, $\Delta_{n}^{2}(k)$ (where $\left.n=1,2,3,4\right)$ are the frequency relaxation parameters of the dynamic structure factor. The parameters $\Delta_{n}^{2}(k)$ are defined as

$$
\Delta_{n}^{2}(k)=\frac{\left\langle\left|W_{n}(k)\right|^{2}\right\rangle}{\left\langle\left|W_{n-1}(k)\right|^{2}\right\rangle},
$$

where $W_{n}(k)$ are the dynamical variables associated with the particle density, longitudinal momentum component, energy density, and so on; the brackets $\langle\cdots\rangle$ denote the ensemble average. This means that the coupling between the density and energy (thermal fluctuations) is included in our theory [5]. The calculation of the frequency relaxation parameters was performed using the molecular dynamics (MD) data and the numerical algorithm in Ref. [5].

Using $S(k, \omega)$ in Eq. (11) gives

$$
\widetilde{C}_{L}(k, \omega)=\frac{\omega^{2}}{k^{2}} \frac{S(k)}{2 \pi} \frac{\Delta_{1}^{2}(k) \Delta_{2}^{2}(k) \Delta_{3}^{2}(k)}{\Delta_{4}^{2}(k)-\Delta_{3}^{2}(k)} \frac{\left[4 \Delta_{4}^{2}(k)-\omega^{2}\right]^{1 / 2}}{\omega^{6}+\mathcal{A}_{1}(k) \omega^{4}+\mathcal{A}_{2}(k) \omega^{2}+\mathcal{A}_{3}(k)} .
$$

In the $\omega \rightarrow 0$ limit and as the consequence of the continuity equation, we have

$$
\lim _{\omega \rightarrow 0} \widetilde{C}_{L}(k, \omega)=0 .
$$

The dispersion relation is [5]

$$
\omega_{c}^{(L)}(k)=\sqrt{\frac{-\mathcal{A}_{1}(k)+\sqrt{\mathcal{A}_{1}(k)^{2}-3 \mathcal{A}_{2}(k)}}{3}} .
$$


The comparison of theoretical $\widetilde{C}_{L}(k, \omega)(13)$ of liquid $\mathrm{Ga}$ at $T=794 \mathrm{~K}$ with inelastic neutron scattering (INS) and MD results shown in Fig. 4 demonstrates good qualitative and quantitative agreement between experiment, modeling, and theory. Theoretical $\omega_{c}^{(L)}(k)(15)$ for the liquid Ga at $T=794 \mathrm{~K}$ is given in Fig. 5, and its comparison to MD data, and INS and IXS experiments similarly demonstrates good agreement. Accordingly, the solid lines in the caption to Fig. 4 refer to the theoretical results in Eq. (13), and the squares in Fig. 5 refer to the theoretical results in Eq. (15).

This shows that the correlation function has the expected low-frequency limit and that the coupling between energy and density is included in our theory.

[1] R. M. Yulmetyev, A. V. Mokshin, P. Hänggi, and V. Y. Shurygin, Phys. Rev. E 64, 057101 (2001).

[2] R. M. Yulmetyev, A. V. Mokshin, P. Hänggi, and V. Y. Shurygin, JETP Lett. 76, 147 (2002).

[3] R. M. Yulmetyev, A. V. Mokshin, T. Scopigno, and P. Hänggi, J. Phys.: Condens. Matter 15, 2235 (2003).
[4] A. V. Mokshin, R. M. Yulmetyev, R. M. Khusnutdinoff, and P. Hänggi, J. Phys.: Condens. Matter 19, 046209 (2007).

[5] A. V. Mokshin and B. N. Galimzyanov, J. Phys.: Condens. Matter 30, 085102 (2018).

[6] T. Bryk, I. Mryglod, and G. Ruocco, Phys. Rev. B 103, 096301 (2021).

[7] R. M. Khusnutdinoff et al., arXiv:2005.00470. 